\title{
Primordial Magnetic Fields, Right Electrons, and the Abelian Anomaly
}

\author{
M. Joyce ${ }^{1,2}$ and M. Shaposhnikov ${ }^{1}$ \\ ${ }^{1}$ Theory Division, CERN, 1211 Geneve 23, Switzerland \\ ${ }^{2}$ School of Mathematics, Trinity College, Dublin 2, Ireland
}

(Received 28 February 1997)

\begin{abstract}
In the standard model there are charges with Abelian anomaly only (e.g., right-handed electron number) which are effectively conserved in the early Universe until some time shortly before the electroweak scale. A state at finite chemical potential of such a charge, possibly arising due to asymmetries produced at the grand unified theory scale, is unstable to the generation of hypercharge magnetic field. Quite large magnetic fields $\left(\sim 10^{22} \mathrm{G}\right.$ at $T \sim 100 \mathrm{GeV}$ with typical inhomogeneity scale $\sim \frac{10^{6}}{T}$ ) can be generated. These fields may be of cosmological interest, potentially acting as seeds for amplification to larger scale magnetic fields through nonlinear mechanisms. Previously derived bounds on exotic $B-L$ violating operators may also be evaded. [S0031-9007(97)03866-0]
\end{abstract}

PACS numbers: 98.80.Cq, 11.10.Wx, 11.30.Fs, 98.62.En

It is usually assumed that the early Universe at temperatures above the electroweak scale and below, say, $10^{12}-10^{16} \mathrm{GeV}$ (depending on the model of inflation) consists of an (almost) equilibrium primordial plasma of elementary particles, in which any long-range fields are absent. One exception is in the context of the problem of generating galactic magnetic fields, which may require the presence of primordial seed magnetic fields which are subsequently amplified by a galactic dynamo mechanism (see, e.g., [1]). The creation of long range magnetic fields requires that conformal invariance be broken in the coupling of the electromagnetic field to gravity [2], and a number of mechanisms based on different ideas about this breaking have been proposed to date [2,3]. In this Letter we argue that there may be a relation between the appearance of magnetic fields in the early Universe and two other, apparently completely unrelated, phenomena: (i) The smallness of the electron Yukawa coupling constant, and (ii) possible lepton asymmetry of the early Universe.

In short, the logic goes as follows. There are three exact conservation laws in the standard electroweak theory. The associated conserved charges can be written as $N_{i}=L_{i}-\frac{1}{3} B$, where $L_{i}$ is the lepton number of $i$ th generation and $B$ is the baryon number. The fourth possible combination, $B+\sum_{i} L_{i}$ is not conserved because of electroweak anomalous processes, which are in thermal equilibrium in the range $100<T<10^{12} \mathrm{GeV}$ [4]. Now, if $h_{e}=0$, where $h_{e}$ is the right electron Yukawa coupling constant, then the electroweak theory on the classical level shows a higher symmetry, associated with the chiral rotation of the right electron field. For the small actual value of the Yukawa coupling $\left(h_{e}=2.94 \times 10^{-6}\right.$ in the MSM), this symmetry has an approximate character. At temperatures higher than $T_{R} \simeq 80 \mathrm{TeV}$ perturbative processes with right electron chirality flip are slower than the expansion rate of the Universe [5], and therefore this symmetry may be considered as an exact one on the classical level at $T>T_{R}$ [6]. [The importance of this symmetry for the consideration of the washout of the grand unified theory (GUT) baryon asymmetry by anomalous electroweak $B$ and $L$ nonconserving reactions was realized in Refs. [5,7,8].] Suppose now that an excess of right electrons over positrons was created by some means at $T>T_{R}$ (e.g., by a GUT mechanism for baryogenesis). Now the right electron number current $j_{R}^{\mu}$ is violated in the minimal standard model (MSM) as described by the anomaly equation

$$
\partial_{\mu} j_{R}^{\mu}=-\frac{g^{\prime 2} y_{R}^{2}}{64 \pi^{2}} f_{\mu \nu} \tilde{f}^{\mu \nu},
$$

where $f(\tilde{f})$ are the $U_{Y}(1)$ hypercharge field strengths (and their duals), respectively, $g^{\prime}$ is the associated gauge coupling, and $y_{R}=-2$ is the hypercharge of the right electron. The number of the right electrons $N_{R}$ therefore changes with the Chern-Simons (CS) number of the hypercharge field configuration as $\Delta N_{R}=\frac{1}{2} y_{R}^{2} \Delta N_{\mathrm{CS}}$ with

$$
N_{\mathrm{CS}}=-\frac{g^{\prime 2}}{32 \pi^{2}} \int d^{3} \vec{x} \epsilon_{i j k} f_{i j} b_{k},
$$

where $b_{k}$ is the hypercharge field potential.

One can now see qualitatively that there is an instability in hot matter with an excess of right electrons toward formation of hypercharge fields with CS number as follows. (The line of reasoning presented here is similar to the consideration of cold fermionic matter with anomalous charges in [9].) The energy density "sitting" in right electrons with a chemical potential $\mu_{R}$ is of the order $\mu_{R}^{2} T^{2}$, and their number density of order $\mu_{R} T^{2}$. On the other hand, this fermionic number can be absorbed by a hypercharge field of order $g^{\prime} k b^{2}$, with energy of order $k^{2} b^{2}$, where $k$ is the momentum of the classical hypercharge field and $b$ is its amplitude. Therefore, at $b>T / g^{2}$ and $k \sim \mu_{R} T^{2} / g^{\prime 2} b^{2}$, the gauge field configuration has the same fermion number as the initial one, but smaller energy. An instability to generation of hypercharge magnetic field, which tends to "eat up" real fermions, results. It is important here that at temperatures $T>T_{R}$ the electroweak symmetry is "restored," and that the U(1) hypercharge magnetic field is massless at that time. (No 
term like $m_{Y}^{2} b^{2}$ is generated in any order of perturbation theory in abelian gauge theory at high temperature [10]; the lattice study in [11] confirmed this expectation for $\mathrm{SU}(2) \times \mathrm{U}(1) \mathrm{EW}$ theory beyond perturbation theory.) If the hypercharge magnetic fields survive until the time of the EW phase transition $(T \sim 100 \mathrm{GeV})$, they will give rise to ordinary magnetic fields because of electroweak mixing. In the rest of this Letter we present quantitative estimates of the (hypercharge) magnetic fields which may be produced by this effect.

Let us discuss first the possible origin and the magnitude of the required right electron number asymmetry $\delta_{R}=$ $e_{R} / s$, where $s=\frac{2}{45} \pi^{2} T^{3} N_{\text {eff }}$ is the entropy density with $N_{\text {eff }}=N_{b}+\frac{7}{8} N_{f}=106.75$ the total effective number of degrees of freedom of the MSM. In principle $\delta_{R}$ produced by out of equilibrium decay at the GUT scale can be as large as $\sim 10^{-2}-10^{-4}$ (for a review see, e.g., [12]). This is quite consistent with the magnitude of the final baryon asymmetry $\delta_{B}$ being that observed since there is no simple general relation between the two numbers. In theories like those discussed in [5,8] with $L$ violating processes at intermediate scales one has $\delta_{B} \sim \delta_{R}$, at least in the case that the $L$ violating processes go out of equilibrium before the $e_{R}$ violating ones come into equilibrium. In [8] the case is considered where the $L$ violation continues for just long enough to reduce the final $\delta_{B}$ to the observed one from an initially larger value fixed by $\delta_{R}$. And, in a simple GUT-like SU(5) in which the charges $N_{i}=L_{i}-\frac{1}{3} B$ are conserved, we can have $\delta_{B}=0$ at the electroweak scale irrespective of the value of $\delta_{R}$ during the time it is effectively conserved. In the rest of this Letter we will simply assume the existence of a primordial density of $e_{R}$, with its chemical potential as a free input parameter, assumed only small enough to be treated perturbatively. We also assume that no hypercharge magnetic fields existed before the right electron excess is generated.

The effect of the anomaly on the gauge field dynamics is given through the term in the effective Lagrangian

$$
\delta \mathcal{L}=\frac{g^{\prime 2}}{4 \pi^{2}} \mu_{R} \epsilon_{i j k} f_{i j} b_{k},
$$

which is obtained by integrating out the fermions at finite chemical potential [13]. It simply describes how winding the gauge fields to give CS number changes the energy of the system because it changes the number of fermions as described by the anomaly equation. Adding this term to the ordinary Lagrangian for the gauge fields leads to the equations of motion

$$
\begin{gathered}
\frac{\partial \vec{H}}{\partial t}+\vec{\nabla} \times \vec{E}=0, \\
\vec{E}=\frac{1}{\sigma}\left(\vec{\nabla} \times \vec{H}+\frac{4 \alpha^{\prime}}{\pi} \mu_{R} \vec{H}\right),
\end{gathered}
$$

where $\alpha^{\prime}=g^{\prime 2} / 4 \pi$. These are simply Maxwell's equations with the additional term due to the anomaly $\left(\propto \mu_{R} \vec{H}\right)$ and the assumption that the total (hypercharge) current is given by $\vec{j}=\sigma \vec{E}$, where $\sigma$ is the conductivity of the plasma and $\vec{E}$ is the (hyper-)electric field. We have also dropped the term $\partial \vec{E} / \partial t$ since (as we will see below) the fields always evolve on a time scale which is much longer than $\sigma^{-1}$. In the expanding Friedman-Rodman-Walker Universe with scale factor $a$, the equations have exactly the same form in conformal time coordinates $\tau=\int a^{-1}(t) d t$, but with the replacements $\mu_{R} \rightarrow \mu_{R} a$ and $\sigma(\propto T) \rightarrow \sigma a$. The fields $\vec{E}$ and $\vec{H}$ are those given by their standard definitions in the conformal frame which will be related to the physical fields at the appropriate point below. We also have the following kinetic equation for $\mu_{R}$ :

$$
\frac{1}{a} \frac{\partial\left(\mu_{R} a\right)}{\partial \tau}=-\frac{\alpha^{\prime}}{\pi} \frac{783}{88} \frac{1}{a^{3} T^{2}} \vec{E} \cdot \vec{H}-\Gamma_{R}\left(\mu_{R} a\right),
$$

in which the first term describes the change in the chemical potential due to the anomaly $(f \tilde{f} \propto \vec{E} \cdot \vec{H})$, and the second the change due to the perturbative processes which flip electron chirality with the rate $\Gamma_{R}=\frac{T_{R}}{M_{0}} T\left(M_{0}=\right.$ $\left.M_{\mathrm{pl}} / 1.66 \sqrt{N_{\mathrm{eff}}} \simeq 7.1 \times 10^{17} \mathrm{GeV}\right)$. The numerical coefficient $\frac{783}{88}$ comes from the relationship between right electron chemical potential and right electron number asymmetry (in terms of which the anomaly is expressed)

$\mu_{R}=\frac{2}{45} \pi^{2} N_{\text {eff }}\left[\frac{783}{88} \delta_{R}-\frac{201}{88} \delta_{1}+\frac{15}{22}\left(\delta_{2}+\delta_{3}\right)\right] T$,

which is obtained from a local thermal equilibrium calculation in the EW theory with three fermionic generations and one scalar doublet, with the conserved charges assumed to be $N_{i}\left(=\delta_{i} s\right)$ and $e_{R}\left(=\delta_{R} s\right)$.

With a Fourier mode decomposition $\vec{H}(\vec{x})=\int d^{3} \vec{k} \times$ $\vec{H}(\vec{k}) e^{-i \vec{k} \cdot \vec{x}}$ with $\vec{H}(\vec{k})=h_{i} \vec{e}_{i}$, where $i=1,2, \vec{e}_{i}^{2}=1$, $\vec{e}_{i} \cdot \vec{k}=0, \vec{e}_{1} \cdot \vec{e}_{2}=0$, the linear equations (4) and (5) become

$$
\begin{aligned}
& \partial_{\tau} h_{1}+\frac{k^{2}}{\sigma a} h_{1}-\frac{4 i \mu|k|}{\sigma} h_{2}=0, \\
& \partial_{\tau} h_{2}+\frac{k^{2}}{\sigma a} h_{2}+\frac{4 i \mu|k|}{\sigma} h_{1}=0,
\end{aligned}
$$

where $\mu \equiv \frac{g^{\prime 2}}{4 \pi^{2}} \mu_{R}$. The mode

$$
\begin{aligned}
h_{2}(\tau, k) & =-i h_{1}(\tau, k) \\
& =-\frac{i}{2}\left[h_{1}(0, k)+i h_{2}(0, k)\right] \exp \left[\lambda_{+}(\tau)\right],
\end{aligned}
$$

where

$$
\lambda_{ \pm}(\tau)=-\frac{k}{\sigma a}\left(k \tau \mp 4 \int_{0}^{\tau} d \tau^{\prime} \mu a\right)
$$

is an unstable mode which is growing at conformal time $\tau$ if $k<4 \mu(\tau) a(\tau)$. It has the property $\vec{E}(\vec{k})=\frac{1}{\sigma a} \times$ $(-|k|+4 \mu a) \vec{H}(\vec{k})$. (The other orthogonal mode decays at any $|k|$.) 
Consider now the approximation in which the chemical potential $\mu$ is a constant. The growing instability starts to develop at $T \sim T_{g}$ where we define $T_{g}$ to be

$$
8\left(\frac{\mu}{T}\right)^{2} \frac{1}{\sigma / T} \frac{M_{o}}{T_{g}}=1
$$

(when the maximally growing mode with $k=2 \mu a$ has begun growing significantly). A necessary requirement for the instability to develop is that $T_{g}>T_{R}$, since if this is not satisfied the second term in (6) will rapidly reduce $\mu$ toward zero. Translated into a minimum value for $\delta \equiv \frac{\mu}{T}$, this requires $\delta>\delta_{\text {crit }}=10^{-6}$ (using $\sigma \approx 68 T$ [14]). For $\delta<\delta_{\text {crit }}$ no nontrivial dynamics result from the presence of such a chemical potential since the unstable modes are frozen on the relevant time scale. If $\delta>\delta_{\text {crit }}$ the evolution of the instability for $T<T_{g}$ will be given by the simple growth factor above, until the time at which the growth becomes significant enough that the first term in (6) is important. To estimate when this is and what the amplitude of the field is at that time it is sufficient to calculate the CS number as a function of time. It is given (per comoving volume) by

$$
\begin{aligned}
n_{\mathrm{CS}}(\tau) & =-\frac{g^{\prime 2}}{32 \pi^{2}}\left\langle\epsilon_{i j k} f_{j k}(\tau) b_{i}(\tau)\right\rangle \\
& \approx-\frac{g^{\prime 2}}{64 \pi^{4}} \int_{0}^{4 \mu a} d k e^{2 \frac{k(4 \mu a-k) \tau}{\sigma a}} k^{2} f(k),
\end{aligned}
$$

neglecting all but the growing mode. We have also taken $\left\langle b_{i}(\vec{k}, \tau) b_{j}^{*}(\vec{l}, \tau)>\left.\right|_{\tau=0}=\delta^{3}(\vec{k}-\vec{l}) \delta_{i j}<b^{2}(k)\right\rangle_{0}, \quad$ assuming translational and rotational invariance of the initial perturbations, and assumed that the perturbations are thermal in origin, with the appropriate normalizations, $\left\langle b^{2}(k)\right\rangle_{0}=\frac{1}{2 k(2 \pi)^{3}} f(k)$, where $f(k)=\left(e^{\frac{k}{T_{0}}}-1\right)^{-1}$ is the bosonic distribution function and $T_{0}$ the temperature at which we define $a_{0}=1$. Defining $\epsilon$ by $\frac{1}{2} y_{R}^{2} n_{\mathrm{CS}} \equiv \epsilon \Delta e_{R} a^{3}$, where $\Delta e_{R}=\frac{88}{783} \frac{\mu_{R}}{T} T^{3}$, i.e., the difference between the right electron density in the initial state and the $\mu_{R}=0$ state, the linear approximation breaks down when $\epsilon \sim 1$. Evaluating the integral in (11) we find $\epsilon \approx 2 \times 10^{-6} \delta \frac{1}{\sqrt{\alpha}} e^{\alpha}$ where $\alpha=T_{g} / T$ with $T_{g}$ as in (10). Thus for a few expansion times after the mode starts growing at temperature $T_{g}$, we have $\epsilon<1$ and the linear approximation is valid. The corresponding physical magnetic field $H_{\text {phy }}$ can be estimated by putting $\left|n_{\mathrm{CS}}\right| \approx \frac{g^{\prime 2}}{16 \pi^{2}} k b^{2}=\frac{1}{2} \epsilon \Delta e_{R} a^{3}$ and using $k b=a^{2} H_{\text {phy }}$, where $k \sim 2 \mu a$ (i.e., assuming the maximal growing mode to dominate). Putting in the numbers this gives a physical magnetic field of strength $H_{\text {phy }} \approx 2 \times 10^{2} \sqrt{\epsilon \delta \frac{k_{\text {phy }}}{T}} T^{2}$ at a physical length scale $k_{\text {phy }}^{-1} \equiv\left(\frac{k}{a}\right)^{-1} \sim \frac{1}{2 \delta T}$. For $\delta \sim 5 \times 10^{-6}$ we are in the linear regime until $T_{R} \sim 80 \mathrm{TeV}$ and at that point therefore have a magnetic field of strength $H_{\mathrm{phy}} \sim 6 \times 10^{26} \mathrm{G}$ $\left(1 \mathrm{GeV}^{2}=1.95 \times 10^{20} \mathrm{G}\right)$ at a length scale of $\sim 10^{5} / T$ (compared to a horizon scale of $\sim 10^{13} / T$ ).

How do the fields evolve for $T<T_{R}$ ? In the case that the linear (i.e., constant chemical potential) approximation is good, one expects that the growth will rapidly turn into decay as $\mu$ is damped. Within a few expansion times the growth will be undone as the maximally growing mode $k \sim 2 \mu a$ now decays with exponent $-\left(k^{2} / \sigma\right) a \tau$. What about the case when this linear approximation breaks down? To treat this case we must analyze the full nonlinear set of Eqs. (4)-(6). We have done this numerically with the simplifying assumption that the distribution of right electron number is homogeneous in space. Then the two linear equations (4) and (5) can be solved exactly for any time dependent $\mu$, the solution inserted in (6), and the averaging over thermal initial conditions performed. The resulting equation is

$$
\begin{aligned}
\frac{\partial \mu a}{\partial \tau}= & \left(\frac{\alpha^{\prime}}{\pi}\right)^{2} \frac{783}{88} \frac{1}{32 \pi^{2} \sigma a^{2} T} \int_{0}^{\infty} d k k^{2} \\
& \times\left\{(k-4 \mu a) \exp \left[2 \lambda_{+}(\tau)\right]\right. \\
& \left.-(k+4 \mu a) \exp \left[2 \lambda_{-}(\tau)\right]\right\},
\end{aligned}
$$

with $\lambda_{ \pm}(\tau)$ given by (9). Our results show that the chemical potential, typical physical momentum of the magnetic field configuration, and the magnetic field energy scale as

$$
\frac{\mu_{R}}{T} \propto \frac{k_{\mathrm{phy}}}{T} \propto \frac{H_{\mathrm{phy}}^{2}}{T^{4}} \propto\left(\frac{T}{T_{g}}\right)^{\frac{1}{2}}
$$

in the range $T_{g}>T>T_{R}$. This behavior can be easily understood qualitatively as follows. As the instability develops, the linear approximation breaks down and $\mu$ starts significantly decreasing. This shifts the growth of modes to longer wavelengths. This procedure continues, growth of any mode eventually turning itself off and increasing the growth coefficient of modes at larger scales. The minimum value of $\mu$ which can be reached at any given time $\tau$ (and, correspondingly, the maximum physical scale for the sourced fields) is simply that given by (10), solved for $\mu$ with $T_{g}$ replaced by the temperature $T(\tau)$; i.e., it is just the minimal chemical potential required to drive a growing mode at that time in the linear approximation. The parametric dependence on the temperature observed follows from the fact that the chemical potential and maximally growing mode trace these values. The dependence of the magnetic field energy follows from the expression we derived in the previous paragraph by setting the CS number of the configuration to cancel the total fermion number, but now taking the appropriate scaling for $k_{\text {phy }}$ itself.

Evolving the system forward from $T_{R}$ to the electroweak scale $T_{\text {ew }}$, we see the damping of the fields for $\delta$ in the linear regime anticipated above, as the perturbative processes erase the chemical potential driving the growth. As $\delta$ increases, however, this damping becomes less efficient, and for $\delta>2 \times 10^{-4}$ we find that the damping has not set in at all by the electroweak scale. The reason for this behavior is also simple. For a mode which evolves in the linear regime, the growth and decay exponents are effectively the same for the modes which grow significantly. Once we enter the nonlinear regime this is 
no longer true, since the maximally growing mode carries the integrated effect of growth until any given time; i.e., it has grown with exponent $k \int \frac{\mu(\tau)}{\sigma} d \tau$ which is much greater than $\frac{k^{2}}{\sigma a} \tau$. Put another way, the mode has been able to grow on a scale significantly larger than the diffusion length for magnetic field at the relevant time, and it takes some time after the end of the growth for the latter scale to catch up and undo the effect of the instability. For $\delta$ in this region we also see that the typical scale of magnetic fields $k$ and value of the chemical potential at $T_{\text {ew }}$ do not depend on initial asymmetry, $\frac{2 k_{\text {phy }}}{T} \simeq \frac{4 \mu}{T} \simeq \frac{10^{6}}{T}$. The amplitude of magnetic field scales as $H \propto \sqrt{\delta}$ and, e.g., for $\delta=10^{-2}$ we find $H \simeq 4 \times 10^{22} \mathrm{G}$. This is as we would expect from the discussion of the scaling above.

What is the ultimate fate of these magnetic fields? Unless some other effect comes into play in the dynamics, the fields will decay. One such effect is turbulence. With the full set of magnetohydrodynamic (MHD) equations (which include the velocity of the fluid which we have neglected) there is a transition to a turbulent regime when the magnetic Reynold's number $R=\sigma L v$ is large [16]. The reason we have evolved the equations to the electroweak scale is that, if the electroweak phase transition is of first order, it serves as a source of turbulence [15]. Since we have here $\sigma \sim 10^{2} T$ and magnetic fields which begin to grow on length scales $L$ up to $\sim 2 \times 10^{6} / T$, we expect to enter the turbulent regime if there are bulk velocities of greater than $\sim 5 \times 10^{-9}$, which are certainly larger that the expected velocity of the bubble walls. A recent study of this phenomenon [16] suggests that the effect of this turbulence is to transfer the magnetic energy to larger length scales, thus evading the Silk argument [17]. If true, the fields generated by the mechanism under discussion may play the role of the seed galactic magnetic fields. Note that the seed fields we obtain at the electroweak scale with the mechanism we have discussed $\left(\sim 10^{22} \mathrm{G}\right)$ are much larger than those generated at bubbles walls $\left(\sim 10^{-2} \mathrm{G}\right)$ which were suggested as seeds for amplification through turbulence in [15]. It is also worth mentioning the particular structure of the magnetic fields appearing because of the abelian anomaly. The CS wave (8) has a nonzero value of $\vec{H} \cdot \vec{\partial} \times \vec{H}$ and thus breaks parity. Could it be that the rotation of galaxies are related to this? Study of the entire set of MHD equations with the additional anomalous terms discussed in this Letter will be required to address this question.

Finally let us mention that the processes we have considered also affect the bounds on the strength of exotic interactions with $B-L$ violation derived from the requirement that GUT baryon asymmetry is not erased by sphalerons $[5,8]$ (which is important if no baryon asymmetry is created at the electroweak scale). If the right electron asymmetry produced at the GUT scale is small enough $(\delta<$ $10^{-6}$ ), then the bounds are obviously not affected since the Abelian anomaly does not play any role. If, on the other hand, $\delta$ is large enough that significant CS number survives remains in the condensate until the electroweak scale, any bound on the strength of exotic interactions can be evaded. Irrespective of the effect of any $B-L$ violation until that point, the remaining CS number will be converted into quarks and leptons carrying net baryon number at the electroweak phase transition. The final baryon asymmetry will depend on the initial value of $\delta$ and the exact strength of the phase transition (which will determine how the $B$ violating processes turn off). Conversely, given detailed knowledge of the phase transition, it will be possible to place an upper bound on the initial value of $\delta$ in the very early Universe, and on the strength of the magnetic fields resulting at the electroweak phase transition.

We are grateful to K. Enkvist, M. Giovannini, A. Kusenko, and L. McLerran for interesting discussions.

[1] S. I. Vainshtein and Ya. B. Zeldovich, Usp. Fiz. Nauk 106, 431 (1972).

[2] M. S. Turner and L. M. Widrow, Phys. Rev. D 37, 2743 (1988).

[3] B. Ratra, The Astronomical J. 391, L1 (1992); A. Dolgov and J. Silk, Phys. Rev. D 47, 3144 (1993); M. Gasperini, M. Giovannini, and G. Veneziano, Phys. Rev. Lett. 75, 3796 (1995); D. Lemoine and M. Lemoine, Phys. Rev. D 52, 1955 (1995).

[4] V.A. Kuzmin, V.A. Rubakov, and M.E. Shaposhnikov, Phys. Lett. 155B, 36 (1985).

[5] B. Campbell, S. Davidson, J. Ellis, and K. Olive, Phys. Lett. 297B, 118 (1992).

[6] In alternative cosmologies such as that considered in M. Joyce, Phys. Rev. D 55, 1875 (1997), in which the expansion rate before nucleosynthesis is different to the standard one, $T_{R}$ can be well below the electroweak scale.

[7] L. E. Ibanez and F. Quevedo, Phys. Lett. 283B, 261 (1992).

[8] J. Cline, K. Kainulainen, and K. Olive, Phys. Rev. Lett. 71, 2372 (1993); Phys. Rev. D 49, 6394 (1994).

[9] V. Rubakov and A. Tavkhelidze, Phys. Lett. 165B, 109 (1985); V. Rubakov, Prog. Theor. Phys. 75, 366 (1986).

[10] E. S. Fradkin, Proc. Lebedev Inst. 29, 1 (1965).

[11] K. Kajantie, M. Laine, K. Rummukainen, and M. Shaposhnikov, Nucl. Phys. B493, 413 (1997).

[12] E. W. Kolb and M.S. Turner, The Early Universe (Addison-Wesley, Reading, MA, 1990).

[13] A. N. Redlich and L.C. R. Wijewardhana, Phys. Rev. Lett. 54, 970 (1985).

[14] M. Joyce, T. Prokopec, and N. Turok, Phys. Rev. D 53, 2930 (1996); G. Baym and H. Heiselberg, Report No. astro-ph/9704214.

[15] G. Baym, D. Bodecker, and L. McLerran, Phys. Rev. D 53, 662 (1996).

[16] P. Olesen, Report No. astro-ph/9610154; A. Brandenburg, K. Enqvist, and P. Olesen, Report No. hep-ph/9608422; Phys. Rev. D 54, 1291 (1996).

[17] J. Silk, Astrophys. J. 151, 459 (1968).

[18] J. Harvey and M. Turner, Phys. Rev. D 42, 3344 (1990); B. Campbell, S. Davidson, J. Ellis, and K. Olive, Phys. Lett. 256B, 457 (1991); W. Fischler, G. F. Giudice, R. G. Leigh, and S. Raban, Phys. Lett. 258B, 45 (1991). 